\title{
DRAMATIC REDUCTION OF DC FIELD EMISSION FROM LARGE AREA ELECTRODES BY PLASMA-SOURCE ION IMPLANTATION*
}

\author{
C. K. Sinclair ${ }^{\dagger}$, H. F. Dylla, and T. L. Siggins, TJNAF, Newport News, VA 23606, USA \\ D. Manos, L. Wu, and T. J. Venhaus, College of William \& Mary, Williamsburg, VA 23187, USA
}

\begin{abstract}
Field emission is one of the principal phenomena limiting the operating voltage of practical electron guns. There is interest in developing photoemission cathode based DC electron guns employing cathode field strengths and cathode-anode voltages well above the present stateof-the-art. Such electron sources could provide high brightness, high average current beams for energy recovered superconducting linear accelerators, for applications in next generation light sources, electron cooling, and electron-ion colliders. We have studied the effect of plasma-source ion implantation on the field emission behavior of large area stainless steel electrodes. Our apparatus allows operation of disc-shaped electrode pairs with $100 \mathrm{~cm}^{2}$ uniform field area to $125 \mathrm{kV}$. The cathode electrode is biased at high voltage, and the anode is electrically isolated, allowing measurement of the field emission current. Electrodes were either mechanically polished, or implanted with nitrogen ions. Two separate ion implanted electrodes have shown negligible field emission at $20 \mathrm{MV} / \mathrm{m}$, and emission between 0.5 and 1.8 $\mathrm{pA} / \mathrm{cm}^{2}$ during multi-hour runs at $30 \mathrm{MV} / \mathrm{m}$. These electrodes show very little conditioning effect.
\end{abstract}

\section{INTRODUCTION}

Photoemission cathodes are able to support high instantaneous current densities, and are a natural choice to produce electron beams bunched directly at the cathode. To achieve the highest brightness, however, it is necessary to mitigate or control the effects of space charge. Thus, the field at the cathode must be large compared to the self field of the bunch, and it is desirable to rapidly accelerate the beam to a relatively high energy. In the case of an injector for a low duty factor accelerator, these requirements are well satisfied by the use of a RF gun. However, for a number of currently envisioned applications, high brightness, high average current CW beams are required [1]. CW operation of RF guns at high field strengths is problematic, so for these new applications it is natural to investigate the possibility of operating DC electron guns with high cathode field strength and high cathode-anode voltage.

Field emission from the cathode electrode and its support structure is the principal phenomenon limiting the maximum electrode field strength and operating voltage of DC electron guns. Field emitted electrons may cause charging, and ultimately breakdown or punch-through of

*Work supported by the USDOE under contract DE-AC05-ER40150 and the Office of Naval Research

‘sinclair@jlab.org the ceramic insulator that supports the cathode electrode. Field emitted electrons may cause melting of materials they strike, and ultimately may result in arcing or breakdown inside the gun structure. Finally, field emitted electrons release gases from materials they strike. These gases may chemically poison high quantum efficiency photocathodes, and also provide a source of residual gas leading to ion back bombardment damage of the cathode.

Field emission from large area DC electrode structures is poorly understood. Models have been developed describing a number of physical phenomena capable of producing high levels of field emission at relatively moderate field strengths [2]. Some of these phenomena have been observed experimentally. However, the fact remains that there are no prescriptions that allow one to confidently choose materials and processes for the construction of DC electron guns that will operate reliably at high voltages and high electrode field strengths. Generally speaking, DC field strengths above about 10 $\mathrm{MV} / \mathrm{m}$ on large area electrodes are problematic in practice, and fields above this level are not normally employed in DC electron guns.

Rather than attempt to gain a fundamental understanding of DC field emission from broad area electrodes, we have designed and constructed a test chamber that allows us to evaluate the field emission performance of various candidate electrode materials and electrode processing techniques. Many field emission measurements have been made on small area electrodes with small cathode-anode gaps. It is difficult in practice to extrapolate these measurements to the performance of real electron guns. Accordingly, our test chamber is designed to test a large electrode area at a relatively high voltage.

Ion implantation is known to produce smooth and hard surfaces on many metals. Unlike surfaces prepared by thin film deposition, ion implanted surfaces are not subject to bonding failure or delamination. Properly prepared, an ion implanted surface will have a uniform chemical composition and work function. It is natural to examine whether the desirable characteristics of ion implanted surfaces lead to improved field emission performance at high field strengths.

\section{THE TEST CHAMBER}

Our test chamber is assembled around a standard stainless steel 6-way vacuum cross with $25 \mathrm{~cm}$ flanges. A ceramic insulator identical to those in use on our $100 \mathrm{kV}$ electron guns isolates the cathode electrode. The triple points of the ceramic are electrostatically shielded, and 
the insulator assembly may be conservatively operated in air at $125 \mathrm{kV}$.

The cathode and anode electrodes are electrically identical. The cathode is mounted on a three inch diameter tube, while the anode is held by gravity on three sapphire balls to provide electrical insulation. Each of these three balls is in turn seated in a precision manual translation mechanism, to permit the anode to be made parallel to the cathode, and the anode-cathode gap to be adjusted. The chamber is pumped by a large differential ion pump. The entire system is ultrahigh vacuum compatible. The system is assembled on a thermally insulating table, which forms the base for an oven, making vacuum bakeout straightforward. Bakeouts were done to $150 \mathrm{C}$. A typical post bakeout pressure in the $10^{-10}$ torr range is achieved. The full system is shown schematically in figure 1.

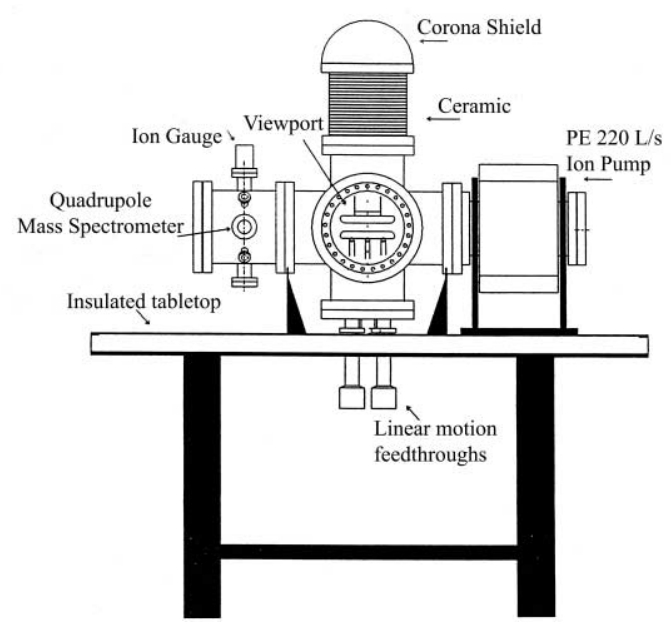

Figure 1: Schematic view of the large area electrode test chamber

The anode and cathode have a quasi-Rogowski profile. The electric field strength calculated by the code POISSON is uniform to within less than $1 \%$ of the central value over a diameter of $12.2 \mathrm{~cm}$, giving a uniform field area over $116 \mathrm{~cm}^{2}$. The anode electrode has additional mass attached to it to assure that it remains seated on the sapphire balls at field strengths up to $35 \mathrm{MV} / \mathrm{m}$. The same anode was used for all tests reported here. It was made of stainless steel, and hand polished with diamond paste to a $1 \mu \mathrm{m}$ surface finish.

All cathode test electrodes were hand polished with diamond paste to a specified surface finish - typically 1 $\mu \mathrm{m}$. Following polishing, they were degreased and cleaned for ultrahigh vacuum. They were transported through room air to and from the ion implantation system, and into the test chamber. The test chamber was located in a concrete vault used for electron gun and high voltage testing. The air for this vault is not filtered, and the environment might best be described as "industrial". The electrodes were blown with dry nitrogen prior to installation in the test chamber, but the likelihood that the electrodes are completely free of dust and particulate matter is nil.

\section{PLASMA SOURCE ION IMPLANTATION}

In plasma source ion implantation (PSII), the work piece is immersed in a plasma of the species to be implanted. The plasma is maintained by an external energy source. Negative high voltage pulses are applied to the work piece, accelerating positive ions from the plasma into it. This technique is well suited for uniformly implanting large surface area three dimensional pieces.

The PSII system we used was developed at the College of William and Mary. The plasma is initiated by a hot filament and a DC glow discharge. RF power was inductively coupled to the plasma by a planar coil. The system has been fully characterized, and is described in detail in the thesis of $\mathrm{L}$. Wu [3]. The system has been operated in two modes. In both cases, the plasma was formed in pure nitrogen gas. In the first case, the RF power was coupled into the plasma through a large area unshielded quartz window. In this case, electrostatic coupling of the antenna to the plasma sputters the quartz window, causing the work piece to become coated with silicon dioxide while simultaneously being implanted by nitrogen. Two stainless steel electrodes, both with a $9 \mu \mathrm{m}$ surface finish, were prepared in this way. In the second case, a Faraday shield was placed between the window and the antenna, minimizing the electrostatic coupling and eliminating the sputter coating. Future studies with this apparatus will thus allow us to differentiate between the effects of ion implantation only, and sputter deposition plus ion implantation.

\section{TEST RESULTS}

We report measurements on four samples. The first is a stainless steel electrode subjected only to hand polishing with diamond paste to a nominal $1 \mu \mathrm{m}$ surface finish. The second is a Ti4V6Al electrode also diamond paste polished to a $1 \mu \mathrm{m}$ finish. The third and fourth are stainless electrodes, both polished to only a $9 \mu \mathrm{m}$ finish, and subjected to the simultaneous $\mathrm{SiO}_{2}$ sputter deposition plus nitrogen implantation treatment.

The performance of the polished stainless steel electrode, shown in figure 2, was not as good as the best we have observed. The polished titanium electrode, shown in figure 3 , behaved reasonably well, and we use titanium alloy electrodes in our electron guns routinely. The sputter coated and ion implanted stainless electrodes, shown in figure 4, show dramatically superior performance to the untreated electrodes. Note the difference in the field emission current scales - three to six orders of magnitude - between these four electrodes. The treated electrodes show essentially no measurable field emission below $20 \mathrm{MV} / \mathrm{m}$, and very small emission even at $30 \mathrm{MV} / \mathrm{m}$. Both of these electrodes were operated at $30 \mathrm{MV} / \mathrm{m}$ for multi-hour periods with no detectable change in the field emission current. We regard these results as very promising. We will continue electrode 
studies for the pure ion implantation, and implantation plus sputter coating cases to assure that we can obtain reproducible results, and to determine which of the two processes produces the best result. If these results stand the test of time, it should be practical to operate DC electron guns with cathode electrode fields of $20 \mathrm{MV} / \mathrm{m}$ or greater. This represents a considerable advance beyond current practice.

I vs E for $5 \mathrm{~mm}$ gap $1 \mu \mathrm{m}$ SST

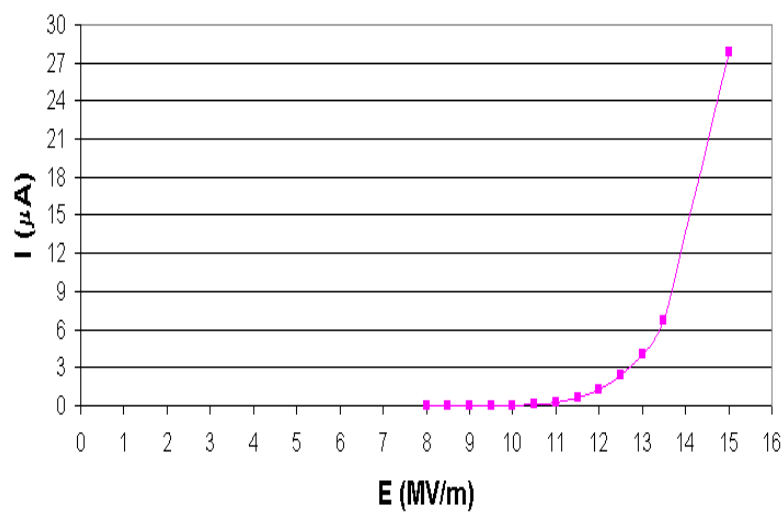

Figure 2: Field emission from a $1 \mu \mathrm{m}$ finish stainless steel electrode

I vs E for $6 \mathrm{~mm}$ gap $1 \mathrm{um} \mathrm{Ti}$

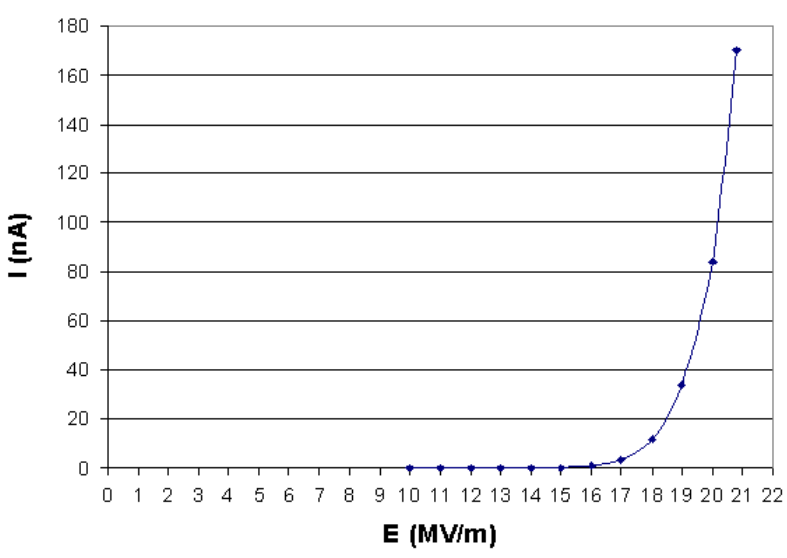

Figure 3: Field emission from a $1 \mu \mathrm{m}$ finish Ti4V6Al electrode
I vs E $4 \mathrm{~mm}$ gap 9um SST Nitrogen Implanted

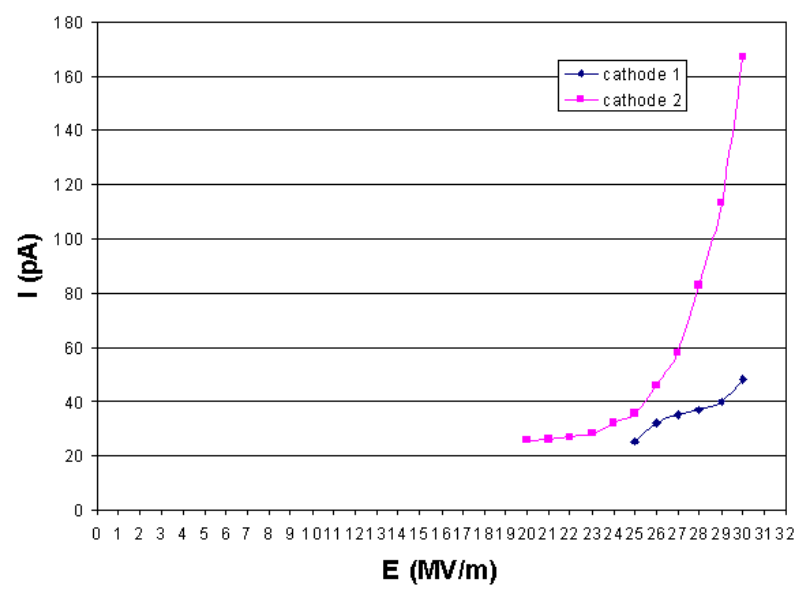

Figure 4: Field emission from two different $9 \mu \mathrm{m}$ finish stainless steel electrodes treated by PSII/PVD

\section{REFERENCES}

[1] See, for example, I. V. Bazarov et al., "The Energy Recovery Linac (ERL) As A Driver For X-ray Producing Insertion Devices", these proceedings.

[2] R. V. Latham, "High Voltage Vacuum Insulation" Academic Press, London, 1995.

[3] L. Wu, Ph.D. thesis, College of William and Mary, October 2000. 